\title{
BASES FISIOLÓGICAS DEL PROGRESO GENÉTICO EN CULTIVARES DEL PROGRAMA REGIONAL DE MAÍZ ${ }^{*}$
}

\author{
Jorge Bolaños ${ }^{2}$
}

\begin{abstract}
RESUMEN
Bases fisiológicas del progreso genético en cultivares del Programa Regional de Maíz (PRM). El desarrollo de germoplasma ha sido uno de los logros más sobresalientes del PRM. El objetivo de este trabajo es determinar parámetros fisiológicos, fenológicos y morfológicos, que expliquen las bases de la elaboración del rendimiento en los cultivares de maíz más importantes del PRM e indagar sobre las bases del progreso genético obtenido. Se establecieron ensayos con nueve cultivares [cuatro híbridos y cinco variedades de polinización libre, (VPLs)] en bloques completos al azar en tres repeticiones a través de 11 di versos ambientes de la región. Los híbridos rindieron consistentemente 1,0-1,5 t/ha por encima de las VPLs a través de todos los ambientes evaluados. La base de estas diferencias parece radicar en un mayor crecimiento diario de la mazorca en los híbridos (2,7 g/ día) que en las VPLs (2,3 g/día). El rendimiento final de cada cultivar es el producto de esta eficiencia por los días de llenado. Dentro de los híbridos y VPLs respectivamente, las diferencias en rendimiento dependieron de la duración de la fase de llenado de grano y no de la eficiencia. Los híbridos HB-85 y CB-HS7 prolongaron seis días más el llenado de grano que P-8916 y H-5, y el Sint.Sequía tuvo tres días más de llenado de grano que las otras VPLs. También se de tectó una fuerte relación negativa entre la duración del llenado y la madurez, sugiriendo que a medida que el genotipo se hace más precoz, pasa menos tiempo produciendo hojas y más tiempo llenando grano. Consistente con estos datos, los híbridos tienen un mayor índice de cosecha que las VPLs. Estos datos indican que prolongar la fase de llenado de grano es un método efectivo para incrementar el rendimiento. Se sugiere que los esquemas de mejoramiento contemplen. estos criterios de selección.
\end{abstract}

\begin{abstract}
Physiological basis of the genetic progress on corn (Zea mays L.) cultivars from the PRM. The germplasm deve10pment has been one of the most outstanding achievements of the PRM (Regional Corn Program). The objective of this test was to understand the physiological basis of genetic gain in the most important corn cultivars from the PRM, by determining the parameters of important physiological, phenological and morphological standards on the elabora-tion of yields. Nine cultivars (5 hybrids and 4 open-pollinated ed varieties OPV's) were evaluated in a Complete Ran-domized block design with 3 replications across 11 diverse environments of the region. The hybrids consistently Clot yielded the OPV' s by 1.0-1.5 t/ha across all the environments. The basis for such differences seemed to rely on the higher daily ear growth-rate of the hybrids $(2.7 \mathrm{~g} /$ day $)$ than of the OPV's ( $2.3 \mathrm{~g} /$ day $)$. The final yield of each cultivar is the product ofthis efficiency by the grain-filling days. Differences in yield among hybrids and OPV's, as germplasm groups, depended on the duration of grain-filling and not on the growth-rate of ears. The CB- HS7 and HB- 85 hybrids took 6 more days of grain-filling than P8916 and H-5. The Sint. Sequia, within the OPV' s, last 3 more days of grain-filling than the others. A large negative relationship was detected between grain-filling lenght and maturity, suggesting that the earlier the genotype, lesser time is invested in producing leaves and more in grain-filling. Consistent with this data, hybrids have a higher harvest index than OPV's. It also suggest that an effective mechanism to increase yield is to extend the grain-filling periodo Current breeding schemes should consider these selec-tion criteria.
\end{abstract}

\section{INTRODUCCIÓN}

El desarrollo de germoplasma de maíz mejorado ha sido la actividad más importante del "Programa Regional de Maíz" (PRM) desde sus inicios hace más de 15 años. En tan sólo los últimos tres años (1989-1991), el PRM ha liberado en la región más de 20 cultivares, incluyendo híbridos y variedades de polinización libre (VPLs), con

\footnotetext{
1 Presentado en la XXXIX Reunión Anual del PCCMCA en Guatemala, América Central. 28 de marzo - 3 de abril, 1993.

2 Agrónomo Regional para Centro América y el Caribe. CIMMYT, Guatemala.

* Publicado en Síntesis de Resultados Experimentales del Programa Regional de Maíz. 1992. V. 4:(1993), p. 11-19. CIMMYT, Guatemala.
} 
tolerancia a diversos factores bióticos y abióticos (Córdova, 1991). Se estima que el PRM ha obtenido ganancias gen éticas cercanos al $2 \%$ por ciclo de selección en sus programas de desarrollo de germoplasma (Córdova, 1991). Esta tasa se compara favorablemente con la obtenida en germoplasma de maíz tropical por Pandey et al., (1987); Paterniani, (1990); Pandey y Gardner, (1992).

Existen varias vías para incrementar la productividad (fracción cosechable) de un cultivo: (a) un incremento en el intervalo de la siembra a la cosecha del cultivo o duración de la fase de llenado de grano; (b) un incremento en la producción de materia seca por unidad de área, y por consiguiente una mayor producción de grano; (c) un incremento en la proporción de la materia seca destinada a la fracción cosechable (mazorca que reflejaría una mejoría en el índice de cosecha; y (d) una mejoría en la tolerancia a alta densidad de población. Esta capacidad también estaría asociada a la capacidad de tolerar estreses ambientales (Fischer y Palmer, 1984; Bolaños y Edmeades, 1993a). Por ejemplo, estudios en germoplasma de maíz tropical con genealogías similares a los cultivares del PRM, indican densidades óptimas por debajo de 50 mil pUha, índices de cosecha menores a 0,40 y menos del $50 \%$ de la duración de la etapa de llenado de grano (Fischer and Palmer, 1984; Paterniani, 1990; Pandey y Gardner, 1992). Ganancias en rendimiento en maíz tropical, caracterizado por ser alto, de excesivo follaje y tardío, han resultado de cambios en la distribución de la materia seca y consecuentes aumentos en el índice de cosecha a medida que la altura de planta, el follaje y la madurez se han reducido (Fischer y Palmer, 1984; Pandey y Gardner, 1992; Bolaños y Edmeades, 1993a). Asociado a estos cambios ha aumentado también la tolerancia a densidad (Fischery Palmer, 1984; Paterniani, 1990).

En contraste, los híbridos de maíz disponibles actualmente en la zona maicera de los Estados Unidos, tienen una densidad óptima de siembra que excede 70000 pl/ha, índices. de cosecha que exceden 0,55 y la fenología del cultivo dedica más del $60 \%$ del tiempo al llenado de grano (Duvick, 1977; Russell, 1984, 1985).
Las ganancias genéticas en los cultivares de maíz de las zonas templadas, ha dependido principalmente del aumento de la duración de la etapa de llenado de grano y mantenimiento del área foliar verde (staygreen) (Duvick, 1977; Russell, 1984, 1985). A pesar que existen caracterizaciones generales sobre maíz tropical, se desconocen las bases fenológicas, morfológicas y/o fisiológicas relacionadas con el rendimiento y posibles vías de ganancias genéticas de los cultivares más importantes del PRM. El objetivo de este ensayo es caracterizar variables estándares explicativos del rendimiento (Fischer y Palmer, 1984; Bolaños y Barreto, 1991; Bolaños y Edmeades, 1993b) en los cultivares más importantes del PRM. Se espera que la información sirva para entender mejor las bases del progreso genético y sus limitaciones.

\section{MATERIALES Y MÉTODOS}

\section{Localidades, Cultivares, Diseño y Manejo Experimental}

Se establecieron un total de 11 ensayos con distintos manejos y en diversos ambientes de América Central, el Caribe y México. Todas las localidades fueron estaciones experimentales normalmente usadas por los investigadores de las instituciones colaboradoras en la conducción del ensayo (Cuadro 1). Algunas de éstas también llevaron tratamientos y condiciones especiales. Los ensayos se establecieron usando semilla proveída por los Programas Nacionales de Maíz y casas comerciales participantes. Se evaluaron nueve cultivares [(cuatro híbridos y cinco variedades de polinización de Sr. denominados (VPLs)] seleccionados con base a su representatividad dentro de los distintos proyectos de mejoramiento del PRM, relevancia comercial y/o histórica (Cuadro 2). Dentro del grupo estaban representados los cultivares que sirven de base a los esquemas de selección regionales del PRM, como el desarrollo de híbridos y tolerancia a sequía, achaparramiento y pudrición de mazorca. 
Cuadro 1. Localidades, fecha de siembra y manejo de los ensayos.

\begin{tabular}{lllll}
\hline & \multicolumn{1}{c}{ País } & Localidad & Fecha & Manejo \\
\hline 01 & Guatemala & Cuyuta & Jun 92 & Optimo \\
02 & Guatemala & San Jerónimo & Jun 92 & Optimo \\
03 & El Salvador & San Andrés & Jun 92 & Optimo \\
04 & Honduras & Omonita & Nov 92 & Optimo \\
05 & Nicaragua & San Cristóbal & Jun 92 & $3 \mathrm{pl} / \mathrm{m}^{2}$ \\
06 & Nicaragua & San Cristóbal & Jun 92 & $7 \mathrm{pl} / \mathrm{m}^{2}$ \\
07 & Panamá & Río Hato & Jun 92 & Optimo \\
08 & R. Dominicana & San Cristóbal & Nov 92 & Optimo \\
09 & México & Tlaltizapán & Jun 92 & Optimo \\
10 & México & Tlaltizapán & Nov 92 & Sequía \\
11 & México & Poza Rica & Nov 92 & Def ic N \\
\hline
\end{tabular}

Cuadro 2. Descripción de los cultivares de maíz evaluados en el ensayo.

\begin{tabular}{|c|c|c|}
\hline Genotipo & Institución & Observaciones \\
\hline HB-85 & $\begin{array}{c}\text { ICTA } \\
\text { Guatemala }\end{array}$ & $\begin{array}{l}\text { Testigo del proyecto de híbridos del PRM, mejor entrada en el ensa- } \\
\text { yo del PCCMCA 1989-91. Blanco, dentado. }\end{array}$ \\
\hline $\mathrm{H}-5$ & $\begin{array}{l}\text { CENTA } \\
\text { El Salvador }\end{array}$ & $\begin{array}{l}\text { Híbrido liberado en } 1960 \text { 's, aún más de } 100 \text { mil ha sembradas } \\
\text { en El Salvador. Testigo histórico. Blanco, dentado. }\end{array}$ \\
\hline P-8916 & $\begin{array}{l}\text { IDIAP } \\
\text { Panamá }\end{array}$ & $\begin{array}{l}\text { Generado y liberado con líneas del PRM, excelente comportamien- } \\
\text { to en ensayos regionales. Amarillo, dentado cristalino. }\end{array}$ \\
\hline CB-HS7 & $\begin{array}{l}\text { Cristiani } \\
\text { Buckard }\end{array}$ & $\begin{array}{l}\text { Híbrido de casa comercial productora de semilla, testigo con genea- } \\
\text { logía asimilar al HB-85. Blanco, dentado.. }\end{array}$ \\
\hline $\begin{array}{l}\text { Sint. Reg. } \\
\text { Pob. } 73\end{array}$ & $\begin{array}{l}\text { CNIGB } \\
\text { Nicaragua }\end{array}$ & $\begin{array}{l}\text { Sintético experimental del último ciclo de selección para acha- } \\
\text { parramiento en Pob. } 73 \text { en PRM. Amplia difusión en Nicaragua } \\
\text { Blanco, dentado. }\end{array}$ \\
\hline Sint. Reg. Sequía & $\begin{array}{l}\text { SRN } \\
\text { Honduras }\end{array}$ & $\begin{array}{l}\text { Sintético experimental del último ciclo de selección para toleran- } \\
\text { cia a sequía en PRM, basada en Tux Seq C6 x B S-19. Blanco, den- } \\
\text { tado. }\end{array}$ \\
\hline $\mathrm{RPMxC} 17$ & $\begin{array}{l}\text { MAG/UCR } \\
\text { Costa Rica }\end{array}$ & $\begin{array}{l}\text { Pob. bajos elección para tolerancia a pudrición de mazorca en PRM, } \\
\text { basada en Tuxp. PB C17 x compuestos criollos de Costa Rica. Blan- } \\
\text { co, dentado. }\end{array}$ \\
\hline CESDA-88 & $\begin{array}{l}\text { CESDA } \\
\text { RepDom } \\
\text { Amarillo, dentado. }\end{array}$ & $\begin{array}{l}\text { Pob. amarilla b/selección a achaparramiento en PRM, muy difun- } \\
\text { dido en Rep. Dom., cruza entre Pob } 36 \text { x Francés }\end{array}$ \\
\hline B-1 & $\begin{array}{c}\text { ICTA } \\
\text { Guatemala }\end{array}$ & $\begin{array}{l}\text { VPL liberada a fines de } 1970 \text { basada en TuxpPBC17, testigo histó- } \\
\text { rico para medir progreso en VPLs. Blanco, dentado. }\end{array}$ \\
\hline
\end{tabular}


Se utilizó un diseño de bloques completos al azar con tres repeticiones (excepto el ensayo 11 que tuvo solamente dos repeticiones) en parcelas de cinco surcos de ocho $\mathrm{m}$ de largo (aunque varió de tamaño en algunas localidades) a una densidad de 5,0 a 5,3 pl/ $/ \mathrm{m}^{2}$ sembrándose dos semillas (para ralear a una planta por golpe) cada $0,25 \mathrm{~m}$ en surcos que variaron de 0,75 a $0,85 \mathrm{~m}$ según la localidad. En las localidades 5 y 6 se establecieron densidades de 3,5 y $7,0 \mathrm{pl} / \mathrm{m}^{2}$ para superimponer también una evaluación de la respuesta a densidad de estos cultivares (Bolaños et al., 1993). Los ensayos se manejaron con $150 \mathrm{~kg}$ $\mathrm{N} / \mathrm{ha}, 40 \mathrm{~kg} \mathrm{P}_{2} \mathrm{O}_{5} /$ iha, control de plagas, enfermedades y malezas, con excepción del ensayo 10 (donde se impuso estrés de sequía suspendiendo el riego del cultivo a los 35 días en un ambiente árido) y 11 (donde se impuso deficiencia de $\mathrm{N}$ usando un bloque manipulado por más de seis años para mantener niveles mínimos de N). La localidad dos también sufrió de estrés reduciendo el rendimiento potencial.

\section{Toma de Datos y Mediciones}

Para las mediciones solamente se usaron plantas en los tres surcos centrales sin usar las dos plantas en los bordos extremos. Entre los cinco y diez días después de la germinación, cuando la hoja número cinco era aún reconocible, esta se marcó en 12 plantas por parcela de una manera que sería reconocible posteriormente (pintura o corte de tijera), y posteriormente se hizo lo mismo con la hoja número diez. Después de la floración, se contó el número final de hojas por planta (NFH), desde la hoja marcada (hoja número diez), así como el número de la posición de la hoja de la mazorca (NHMZ), el número de ramas primarias en la espiga (NRPE), el largo y ancho máximo de la hoja de la mazorca, y el número de hojas verdes por debajo de la mazorca (NHV).

Antes de la floración y después que el $10 \%$ de las espigas eran visibles, se visitó la parcela cada dos o tres días y se determinó la fecha de floración masculina y femenina (50\% de floración DAFM y DAFF). El intervalo entre la antesis y la emisión de los estigmas se calculó como la diferencia entre DAFF y DAFM. Con el mismo procedimiento de visitas continuas antes de la cosecha, se determinaron también los días al 50\% de madurez fisiológica (DMF), y se observó la presencia de capa negra en plantas de los surcos bordo (se hizo sólo en 4 localidades). La duración de llenado de grano, se calculó como la diferencia entre DMF-DAFF.

A la cosecha, 12 plantas al azar se cosecharon para biomasa total y peso de mazorca. El índice de cosecha (IC) se calculó como la proporción de grano en la biomasa total. Todas las plantas restantes en la parcela útil (10$12 \mathrm{~m}^{2}$ ) se cosecharon a mano y se determinó el peso de campo de las mazorcas. En 12 mazorcas al azar, se determinó el índice de desgrane, el número de hileras por mazorca y el número de granos en una hilera para estimar el número total de granos por mazorca. Se determinó el peso y humedad de 200 granos. El rendimiento de grano se uniformizó a un contenido de $15 \%$ de humedad. Se calcularon los componentes de rendimiento estándares (Bolaños y Barreta, 1991). La eficiencia de llenado de grano de la mazorca, o el rendimiento efectivo por día de llenado se calculó al dividir el peso promedio de grano por planta (g/planta) entre la duración del llenado de grano.

\section{Estudio del Desarrollo de la Línea de Leche durante la Maduración del Grano}

En el ensayo 9 (Tlaltizapán), se efectuó un estudio para caracterizar el desarrollo de la línea de leche del grano durante su maduración. La evaluación se inició 45 días después de la floración y en intervalos de tres a cuatro días, hasta llegar a la madurez fisiológica. Se cosecharon cuatro mazorcas en los surcos laterales de cada parcela y se usaron dos granos del centro de cada mazorca, para calificar el desarrollo de la línea de la leche con base en una escala de: 0- para granos aún sin línea de leche (todo lechoso); I-para granos con la línea en el 25\% superior del grano; 2-para granos con la línea entre el 25 y $50 \%$ superior del grano; 3-para granos con la línea entre el 25 y $50 \%$ inferior del grano; 4-para cuando la línea ya no es visible pero aún se puede exprimir leche; y 5-para la presencia de capa negra cuando no se puede exprimir leche (Bolaños y Edmeades, 1993b). Las mazorcas fueron 
posteriormente desgranadas para determinar el porcentaje de humedad y peso de grano.

\section{Análisis y Presentación de Datos}

Se efectuó análisis de varianza de los datos por cada localidad individual y combinado a través de las todas las 11 localidades. Se hicieron contrastes ortogonales entre híbridos y VPLs. Se utilizó el método de estabilidad de Eberhart y Russell (1966).

\section{RESULTADOS Y DISCUSION}

\section{Rendimiento a través de las 11 localidades}

A través de las 11 localidades, los híbridos rindieron con si stente mente por encima de las VPLs, las diferencias aumentaron con el potencial ambiental (Figura 1). Debido a esto, se detectó una interacción significativa genotipo por ambiente en rendimiento, pero sin detectar cruzamiento significativo entre los cultivares.

El híbrido CB-HS7 superó a todas las demás entradas, mostrando rendimiento superior aún en las localidades bajo estrés de sequía y deficiencia de $\mathrm{N}$ (locs 10 y 11). Los híbridos HB-85 y P-8916 ocuparon el segundo y tercer lugar, respectivamente. El híbrido H-5 mostró el comportamiento más pobre entre los híbridos, y en muchos de los casos fue superado por las VPLs. Dentro de las VPLs, el Sint. Sequía rindió consistentemente por encima de las otras (más de 0,4 t/ha) y RPMxC17 mostró el comportamiento más pobre. Los cultivares B-1, Cesda-88 y el Sint. P73 tuvieron un comportamiento similar en rendimiento a través de los ambientes evaluados (Figura 1). Una comparación entre híbrido s y VPLs (excluyendo el H-5 y RPMxCl7 debido a su comportamiento pobre) muestra claramente la superioridad de los híbridos a través de todos los ambientes evaluados (Figura 1). Aún en ambientes con potencial de rendimiento inferior a 1,0 t/ha, los híbrido s superan a las VPL's en más de 0,3 t/ha, y esta diferencia aumenta a casi 1,5 t/ha en ambientes con potencial de rendimiento de 6,0 t/ha. Los parámetros de regresión del modelo Eberhart y Russell (1966) se

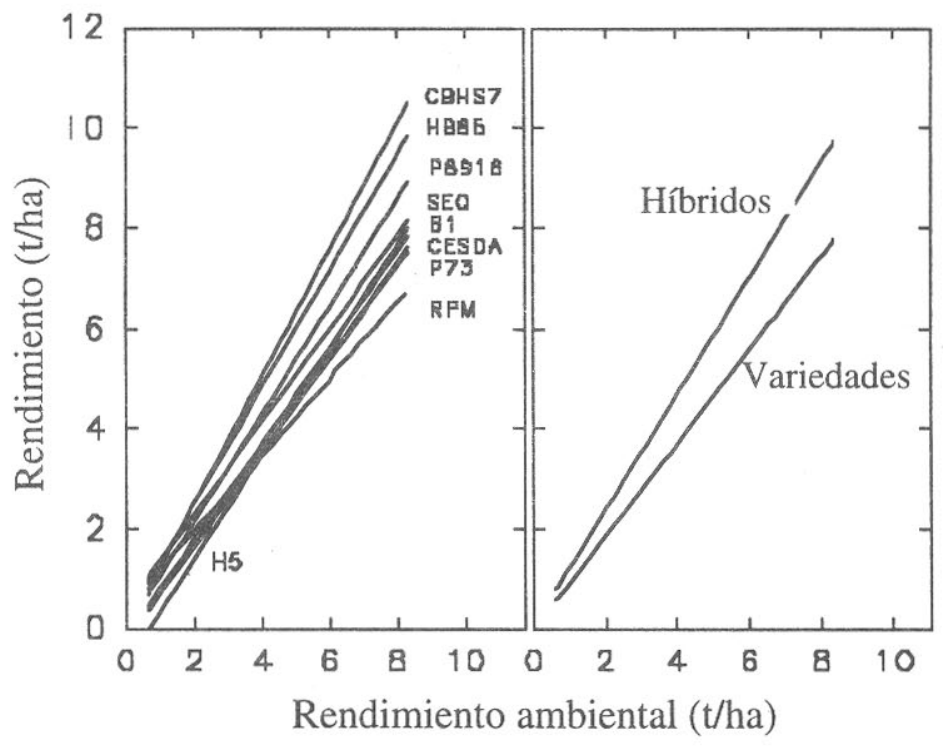

Figura 1. Líneas de regresión entre (a) el rendimiento de los nueve cultivares evaluados y el rendimiento ambiental, definido como el promedio de todos los cultivares en cada localidad; y (b) el rendimiento promedio de los híbridos (ent 1,3,4) y las VPLs (ent 5,6,8,9), contra el rendimiento ambiental. 
presentan en el Cuadro 3. Como se puede notar, en promedio los híbridos tienen coeficientes de regresión (pendientes) de 1,16, mientras que las VPLs de 0,93.

\section{Parámetros Fisiológicos, Fenológicos y Morfológicos en los Cultivares del PRM}

El Cuadro 4, presenta las medias y descriptores estadísticos de los parámetros fisiológicos, fenológicos y morfológicos evaluados en los cultivares a través de los 11 ambientes. Por razones de espacio, no se presentan los análisis individuales por localidad. En promedio, los híbridos rindieron 1,1 t/ha más que las VPLs, principalmente debido a un mayor peso de mazorca $(17 \mathrm{~g} /$ mazorca más que las VPLs), un mayor número de granos por mazorca (48 granos/mazorca más que las VPLs debido a 1,2 hilera adicional por mazorca, pero no a un mayor número de granos por hilera), un mayor número de' mazorcas por área (5000 mazorcas/ha adicionales), y un mayor índice de cosecha (36\% vs $33 \%)$.

Las variables fenológicas y morfológicas (número final de hojas, número de la hoja mazorca, número ramas primarias de espiga, tamaño de la hoja de la mazorca, etc.) muestran pocas diferencias significativas entre los híbridos y las VPLs como grupos de germoplasma.

Es de notar también la superioridad de los híbridos, en especial CB-HS7 y HB-85, en comparación con las VPLs en algunas características agronómicas. Los híbridos mostraron $13 \%$ menos pudrición de mazorcas y $11 \%$ menos de acame que las VPLs (Cuadro 4). Muchas de las VPLs mostraron altos porcentajes de acame y pudrición de mazorca. El cultivar RPMxCI7, con supuesta tolerancia a pudrición de mazorca, y población base del proyecto del PRM para tolerancia a pudrición de mazorca, mostró uno de los porcentajes más elevados.

Sin embargo, hay diferencias notables en algunos cultivares. Por ejemplo, los híbridos H-5 y P-8916, son más tardíos que los otros cultivares, tienen el mayor número final de hojas, mayor altura de planta, menor índice de cosecha y menor duración de llenado de grano dentro de los híbridos. El P-8916 tiene también el mayor número de ramas primarias de espiga. El Sint. Sequía, basado en Tuxpeño C6 Sequía, tiene historia de selección para sincronía floral bajo estrés. Estudios por Bolaños y

Cuadro 3. Coeficiente de regresión e intercepto de regresión entre rendimiento de cada cultivar versus el potencial ambiental) de nueve cultivares a través de 11 localidades.

\begin{tabular}{lccc}
\hline Genotipo & $\begin{array}{c}\text { Coeficiente } \\
\text { Regresión }\end{array}$ & Intercepto & $\mathbf{r}^{2}$ \\
\hline HB-85 & 1,15 & 0,19 & 0,98 \\
H-5 & 1,04 & $-0,68$ & 0,97 \\
P-8916 & 1,07 & 0,02 & 0,99 \\
CB-HS7 & 1,26 & 0,01 & 0,98 \\
Sint. Pob 73 & 0,94 & $-0,22$ & 0,99 \\
Sint. Sequía & 0,92 & 0,46 & 0,99 \\
RPM x C17 & 0,74 & 0,49 & 0,96 \\
Cesda & 0,92 & $-0,13$ & 0,98 \\
B-1 & 0,96 & $-0,13$ & 0,97 \\
Híbridos & 1,16 & 0,07 & 0,99 \\
VPLs' $^{\prime}$ & 0,93 & $-0,01$ & 0,99 \\
\hline
\end{tabular}

1 El promedio de híbridos excluye entrada 2 y el de las VPLs excluye la entrada 7. 


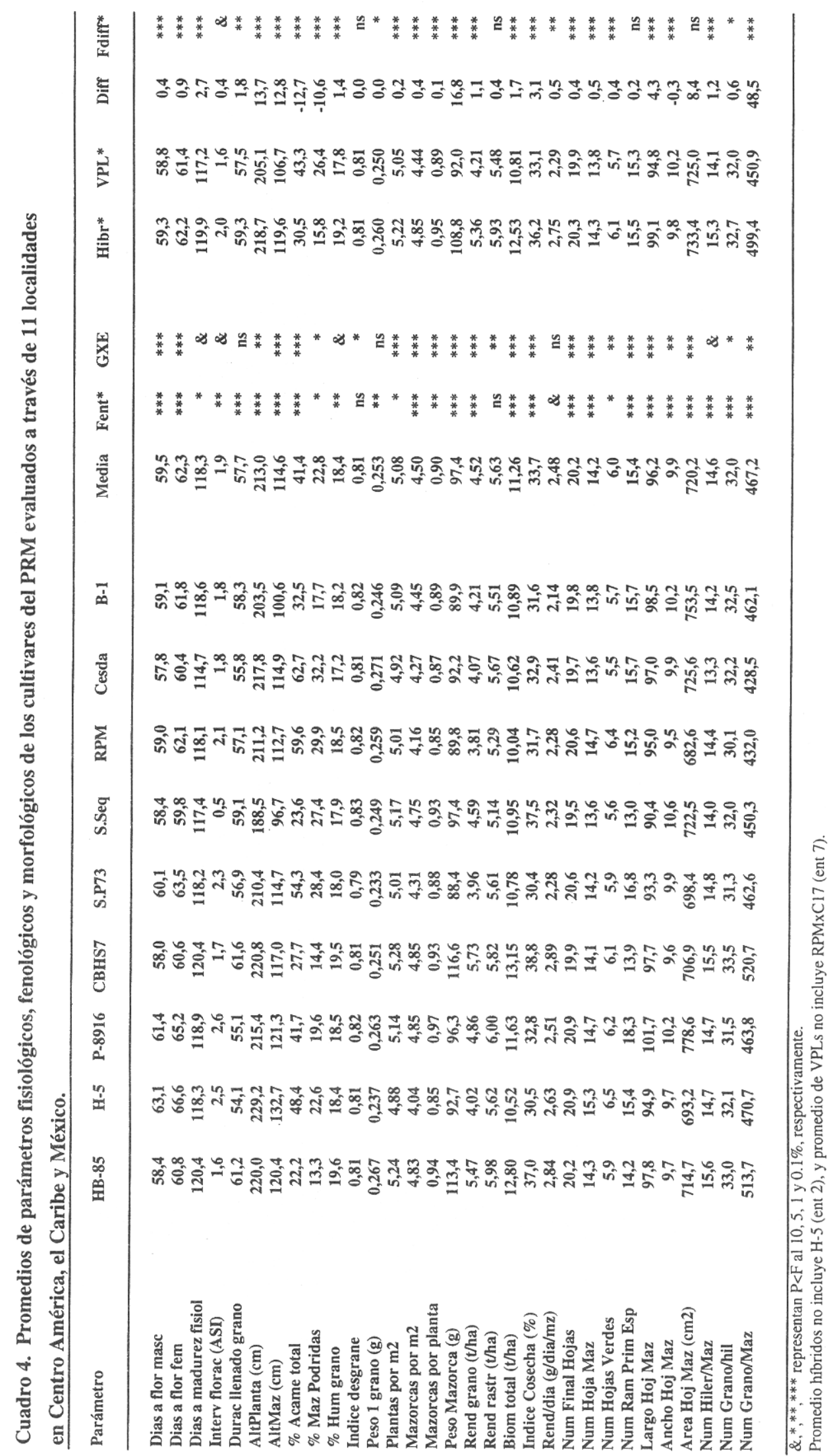


Edmeades (1993a) mostraron una correlación entre la sincronía floral bajo estrés y una mayor distribuición de la materia seca hacia la mazorca. Este cultivar tiene el menor número de ramas primarias de espiga, la menor altura de planta, la mejor sincronía floral, el mayor número de mazorcas por planta e índice de cosecha, en las VPLs. El hecho que el Sint. Sequía rinde consistemente superior a las otras VPLs, confirma los resultados obtenidos por Bolaños y Edmeades (1993a) sobre la eficacia de la selección bajo estrés para el mejoramiento en maíz tropical. El B-1 representa germoplasma con genealogía similar al Sint. Sequía liberado a fines de los 1970's. Comparaciones entre ellos pueden ofrecer pautas de las ganancias gen éticas adquiridas en los últimos años con un esquema de selección bajo estrés (Bolaños y Edmeades, 1993a).

\section{Bases Fisiológicas del Rendimiento}

El rendimiento final de cada cultivar es el producto de la tasa diaria del llenado de grano por el total de días de llenado. La Figura 2 (a,b,c,) muestra la relación entre rendimiento, tasa de crecimiento de la mazorca por día de llenado, y la duración del llenado de grano para los distintos cultivares del PRM. A pesar de grandes diferencias en rendimiento y duración del llenado entre los híbridos y las VPLs, es sorprendente la similaridad en la tasa de crecimiento de la mazorca por día dentro de cada grupo de germoplasma (Figura 2b). Los híbridos distribuyen cerca de $2,7 \mathrm{~g}$ a la mazorca por cada día de llenado de grano, mientras que las VPLs solamente 2,3 g/ día (este número solamente incluye el crecimiento del grano, excluyendo el olote y las hojas de la mazorca). Es importante recalcar la similitud en esta eficiencia dentro de cada grupo de germoplasma, ya sea dentro de híbridos o dentro de las VPLs. Estos datos, si se confirman también en otros cultivares, sugieren que quizás la base fisiológica de la heterosis obtenida en la generación de híbridos está relacionada con su eficiencia del llenado de grano.

Dentro de los híbridos, el progreso genético obtenido a la fecha parece descansar en un alargamiento efectivo del llenado de grano y no de cambios en la eficiencia. Los híbridos CB-HS7 y HB-SS llenan grano seis días

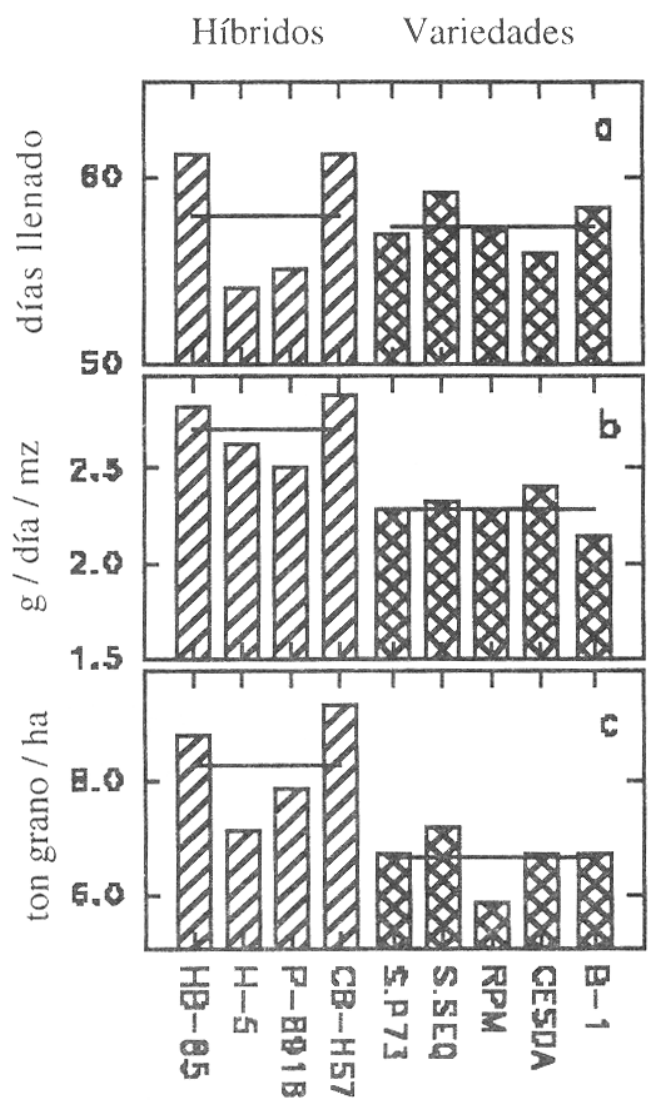

Figura 2. Relación entre (a) duración del llenado de grano en días, (b) el crecimiento diario del grano de la mazorca por cada día de llenado (g/día), y (c) el rendimiento por hectárea (ton/ha) de los cultivares evaluados del PRM. Los datos son de San Jerónimo (Guatemala), Río Hato (Panamá) y Tlaltizapán (México) durante 1992.

más que el R.S y el P-8916. El H-5 refleja el progreso genético existente en la región en los 1960' s, cuando fue liberado, pero aún se siembran más de 100 mil ha por año. Sin embargo, el híbrido P-8916 aún tiene características de madurez tardía, excesivo follaje, espiga grande, duración corta de llenado de grano y bajo índice de cosecha. Estos datos son consistentes con los ensayos uniformes del PCCMCA, conducidos anualmente por el PRM, donde los híbridos amarillos rinden consistentemente por debajo de los blancos (Quemé y Fuentes, 1992; Urbina, 1993). 
El rendimiento inferior de las VPLs en comparación con los híbridos depende de una menor eficiencia de llenado de grano y no solamente de la duración del llenado. Todas las VPLs tienen duraciones de llenado de grano entre 56 y 59 días con pocas diferencias entre sí (Fig. 2a). El Sint. Sequía sobresale por tener la mayor duración de llenado de grano.

Una gráfica entre la duración del llenado de grano, días a floración y número final de hojas muestra una relación negativa significativa entre madurez, número final de hojas y el llenado de grano (Figura 3a. 3b). Estos datos sugieren, por lo menos dentro de este grupo de cultivares, que el aumento en la duración del llenado de grano está relacionado con el proceso de una selección efectiva para maíces más precoces en días a floración, pero con alto rendimiento. Muchos autores han encontrado que los maíces precoces son más eficientes con mayores índices de cosecha (Paterniani, 1990; Pandey and Gardner, 1990). Los resultados indican que prolongar la fase de llenado de grano parece ser un método efectivo para incrementar el rendimiento. Asumiendo una población de 50000 mazorcas/ha y una tasa promedio de llenado de 2,5 $\mathrm{g} /$ día, se puede estimar que el rendimiento aumenta en $125 \mathrm{~kg} / \mathrm{ha}$ por cada día adicional de llenado de grano.

Es poco probable que víveros de selección con más de 200 entradas, con varias repeticiones, puedan examinar en detalle la duración del llenado de grano directamente. Datos de humedad de grano y calificaciones del desarrollo de la línea de leche tomados en el ensayo 9 , muestran una correlación importante entre el desarrollo de la madurez fisiológica y la humedad de grano independiente del cultivar (Figura 4a,b).

Los datos muestran que la humedad de grano puede ser un fácil indicador para monitorear la madurez fisiológica (alcanzada con calificación de 4,5 en la escala usada). La capa negra parece formarse cuando la humedad del grano se acerca al 30\% (Fig. 4a), lo cual ocurre de 60 a 70 días después de la floración femenina, según el cultivar (Fig. 4b).
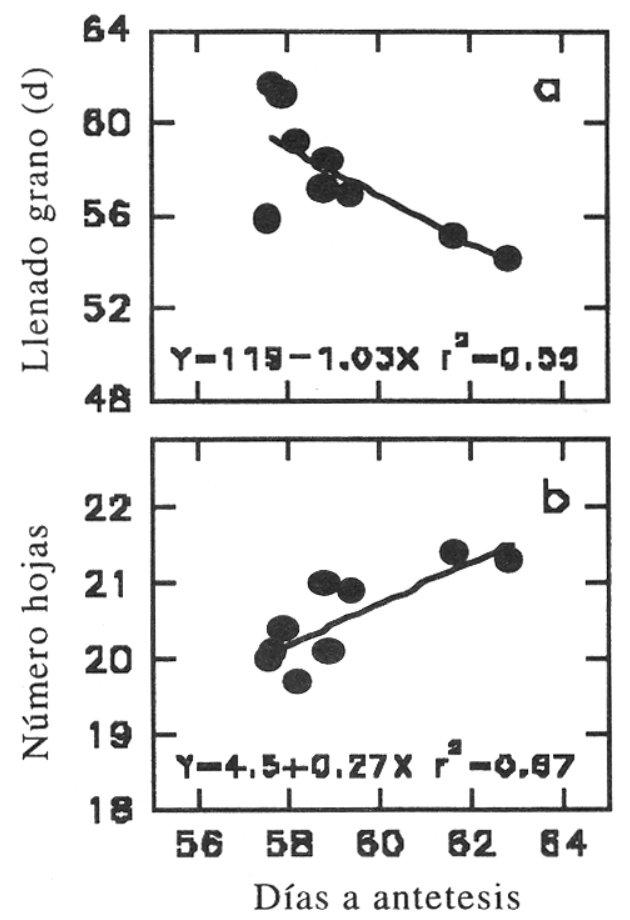

Figura 3. Relación entre la duración del llenado de grano (días) y (a) el número final de hojas, y (b) días a la floración masculina para los cultivares del PRM. Los datos son de San Jerónimo (Guatemala), Río Hato (Panamá) y Tlaltizapán (México) durante 1992.

Cosechando cerca a la madurez fisiológica, o sea, con humedad de grano alrededor de $30 \%$, y con el análisis juicioso de los datos de madurez (días a floración), de humedad de grano (para identificar llenado corto o llenado largo) y de rendimiento (tamaño grande de mazorcas reflejando altas tasas de llenado), se puede integrar un índice para identificar materiales precoces, con largo llenado de grano y alto rendimiento. Es muy factible integrar estos criterios en los programas actuales de selección dentro del PRM, ya sea en los esquemas de selección recurrente o los de endocriamiento y generación de líneas para su posterior hibridización.

\section{CONCLUSIONES}

1. Dentro de los cultivares del PRM evaluados, los hí- 


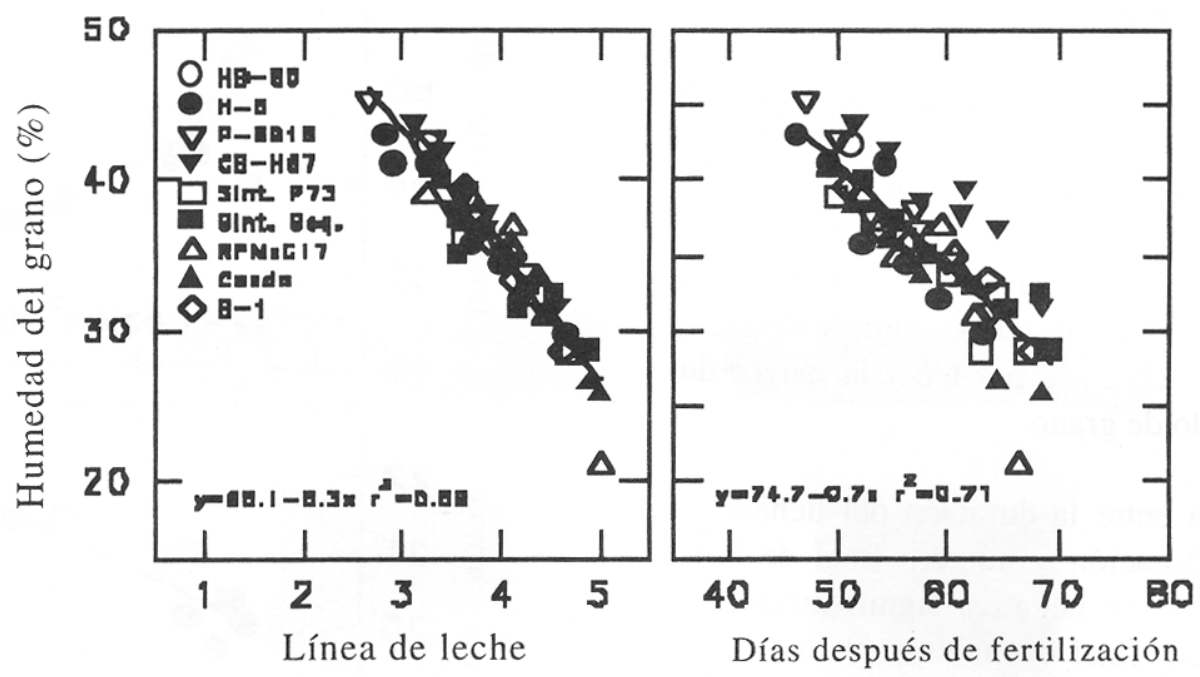

Figura 4. Relación entre humedad del grano y: (a) una calificación visual de la línea de leche (escala de 0-5, 5 siendo (a, b) madurez fisiológica); y (b) tiempo después de la floración femenina (días), durante la fase del lenado de grano para los cultivares del PRM. Los datos son de Tlaltizapán (México) durante 1992.

bridos rinden consistentemente de 1,0 a $1,5 \mathrm{t} / \mathrm{ha}$ por encima de las VPLs, aún en ambientes con bajo potencial de rendimiento.

2. Los híbridos CB-HS7 y HB-85 mostraron el mejor comportamiento a través de todos los ambientes muestreados. Dentro de las VPLs, el Sint. Sequía superó a las demás, en particular en ambientes con estrés de sequía y baja fertilidad. El híbrido H-5, liberado en los 1960' s, fue superado por la mayoría de las VPLs, y el cultivar RPMxC17 tuvo un comportamiento muy pobre. Las VPLs restantes, B-1, Cesda-88 y el Sint. P73 mostraron un comportamiento similar.

3. Las diferencias en rendimiento entre los distintos cultivares se deben a una combinación de mejor eficiencia de llenado (híbridos versus VPLs) y una mayor duración de la fase de llenado de grano. La mazorca crece 2,7 g/día de llenado en los híbridos, pero solamente 2,3 g/día en las VPLs. Los híbridos CBHS7 y HB-85 prolongan el llenado de grano más de seis días que los demás cultivares. El rendimiento final de cada cultivar es el producto de esta eficiencia por los días de llenado.
4. Se detectó una fuerte relación negativa entre la madurez y la duración del llenado de grano, sugiriendo que a medida que el genotipo se hace más precoz pasa menos tiempo produciendo hojas y más tiempo llenando el grano. Se recomienda que los esquemas de mejoramiento del PRM contemplen estos criterios de selección. La humedad de grano puede servir como indicador fácil para identificar la duración del llenado de grano en los programas de mejoramiento.

\section{AGRADECIMIENTOS}

Se agradece a Carlos Pérez, José Luis Quemé, Salvador Castellanos, Fidencio Guerra, Luis Brizuela, Pedro Campos, Róger Urbina, Marvin Obando, Ismael Camargo, Alfonso Alvarado, Rodolfo Pierre, Richard Ortiz, Reneé Lafitte, Greg Edmeades, Mariane Bazinger y muchos otros, por conducir estos ensayos en las distintas localidades y a Edith Tello por la paciente labor de entrada de datos. 


\section{LITERATURA CITADA}

BOLAÑOS, J.; BARRETO, H.. 1991. Análisis de los componentes de rendimiento de los ensayos regionales de maíz de 1990. En: Análisis de los Resultados Experimentales del PRM 1990, CIMMYT, Guatemala. V. 2, pp. 9-27.

BOLAÑOS, J.; EDMEADES, G. O. 1993a. EightcycJes of selection for drought tolerance in tropical maize. 1. Responses in yield, biomass and radiation utilization. Field Crops Res. 31 :233-252.

BOLAÑOS, J.; EDMEADES, G. O. 1993b. La fenología del maíz. p xx-yy. En: Sintesis de Resultados Experimentales del PRM 1992, Vol. 4, CIMMYT, Guatemala.

BOLAÑOS, J.; OBANDO, M.; URBINA, R.; MENDOZA, M. 1993. Respuesta a densidad de los cultivares del PRM. En: Síntesis de Resultados Experimentales del PRM 1992, V. 4, CIMMYT, Guatemala. p. 20-26.

CORDOVA, H. 1991. Desarrollo y mejoramiento de germoplasma para resistencia a factores adversos bióticos y abióticos y producción de semillas: Estrategias y logros 1986-1991. p. 1-15. En: Desarrollo y Mejoramiento de Germoplasma para Resistencia a Factores Adversos Bióticos y Abióticos 1990, CIMMYT, Guatemala.

DUVICK, D. N. 1977. Genetic gains of grain in hybrid maize yields during the past 40 years. Maydica 22:187-196.

EBERHART, S. A; RUSSELL, W. A 1966. Stability parameters forcomparing varieties. Crop. Sci. 6:36-40.
FISCHER, K. S.; PALMER, A F. E. 1984. Tropical maize. p. 213-248. In: P.R. Goldsworthy and N.M. Fisher (Eds.) The Physiology of Tropical Crops, John Wiley and Sons, New York.

PANDEY, S.; GARDNER, C. O. 1992. Recurrent selection for population, variety and hybrid improvement in tropical maize. Adv. Agron. 48:1-87.

PANDEY, S.; DIALLO, A O.; ISLAM, T. M. T.; DEUTSCH, J. 1987. Response to full-sib selection in four medium maturity maize populations. Crop Sci. 27:1241-1245.

PATERNIANI, E. 1990. Maize breeding in the tropics. Critical Rev. Plant Sci. 9(2): 125-154.

QUEMÉ, J. L.; M. FUENTES. 1992. Evaluación de hibridos de maíz de grano blanco y amarillo en diferentes ambientes de México, Centro América, el Caribe y Venezuela. p. 1130. En: Sintesis de los Resultados Experimentales del PRM 1991, Vol. 3, CIMMYT, Guatemala.

RUSSELL, W. A 1984. Agronomic performance of maize cultivars representing different eras of breeding. Maydica, 29:375-390.

RUSSELL, W. A. 1985. Evaluations for plant, ear and grain traits of maize cultivars representing seven years of breeding. Maydica,30:85-96.

URBINA, R. 1993. Evaluación de hibridos de maíz de grano blanco y amarillo en ambientes de Centro América, Panamá, el Caribe y México. p. 27-38. En: Sintesis de los Resultados Experimentales del PRM 1992, Vol. 4, CIMMYT, Guatemala. 